\section{OP19 HOW MUCH DOES AREA LEVEL DEPRIVATION CONTRIBUTE TO TOTAL VARIATION IN AGE AT DEATH?}

${ }^{1} \mathrm{R}$ Seaman*, 'T Riffe, ${ }^{2} \mathrm{H}$ Caswell. 'Max Planck Institute for Demographic Research, Rostock, Germany; ${ }^{2}$ University of Amsterdam, Amsterdam, The Netherlands

\subsection{6/jech-2018-SSMabstracts. 19}

Background Mortality inequalities demonstrate a double burden: the most deprived socioeconomic groups experience the lowest average age of death and the highest variation in age at death. Two processes generate variation in age at death: individual stochasticity (within-group variance) and heterogeneity (between-group inequality). No known research has quantified how much area level deprivation (between-group inequality) has contributed to total variation in age at death or if this has changed over time. We address this research gap using population and mortality data for the entire population of Scotland stratified by a validated measure of area-level deprivation covering 19812011.

Methods Census population estimates, and mortality records were matched with the Carstairs score. Life tables by year, sex and population weighted deprivation quintiles (20\%) were constructed. Lifespan variation was calculated and transformed into the standard deviation to allow interpretation in number of years. Markov Chain methods were used to decompose the total lifespan variation in to the within deprivation-group and between deprivation-group component at each Census year, for males and females separately.

Results Total variation in age at death for males in Scotland increased between 1981 and 2011. For females there was limited change. Variation stagnated for the most deprived but decreased for the least deprived. Although the betweengroup component of variation in age at death is small the relative proportion doubled between 1981 and 2011 for males and females, from $1 \%$ to $3 \%$ and from $1 \%$ to $2 \%$ respectively. To give context, the proportion of total variance due to between-sex differences in age at death decreased from 4\% in 1981 to $2 \%$ in 2011, meaning that inequalities in deprivation are comparable with sex differences when seeking to explain variation in age at death. Analysis repeated for population weighted deciles produced greater increases: quintiles are the preferred analytical grouping in Scotland. Analysis using the most and least deprived quintiles only showed that the contribution from deprivation was 4 times higher than when using all quintiles. Results reported used standard Markov stationary mixture assumptions and were unchanged when allowing empirical age-specific mixing distributions.

Conclusion Relative contributions from area level deprivation to total variance in age at death doubled in Scotland between 1981 and 2011 revealing the changing nature of mortality inequalities. These findings are relevant for governments deciding whether to invest in social policies that intervene at the areal level or at the individual level.

\section{OP20 A COMPARISON OF INEQUALITIES IN ALL-CAUSE AND CAUSE-SPECIFIC MORTALITY BY WEALTH AND INCOME: A REGISTRY-BASED COHORT STUDY OF THE SWEDISH POPULATION}

${ }^{1} \mathrm{SV}$ Katikireddi ${ }^{*},{ }^{2} \mathrm{C}$ Niedzwiedz, ${ }^{1} \mathrm{R}$ Dundas, ${ }^{1} \mathrm{AH}$ Leyland, ${ }^{3} \mathrm{~N}$ Kondo, ${ }^{4} \mathrm{M}$ Rostila. ${ }^{1} \mathrm{MRC} /$ CSO Social and Public Health Sciences Unit, University of Glasgow, Glasgow, UK; ${ }^{2}$ Institute of Health and Wellbeing, University of Glasgow, Glasgow, UK; ${ }^{3}$ School of Public Health, University of Tokyo, Tokyo, Japan; ${ }^{4}$ Centre for Health Equity Studies, Stockholm University, Stockholm, Sweden

\subsection{6/jech-2018-SSMabstracts.20}

Background Wealth inequalities are increasing in many countries but their impact on health, and particularly mortality, has been little studied. Existing studies have focused on specific age groups using survey data, with no studies investigating mortality inequalities by wealth across adulthood. We investigated the relationship between individual wealth and mortality across the adult life course in the Swedish population.

Methods We studied the entire Swedish adult population in 1990 using national registries, with follow-up for 19 years. Our main exposure of interest was the amount of tax paid in a historical tax on wealth, calculated using a comprehensive assessment including real estate (value in prior year minus outstanding mortgages), high value personal items (e.g. cars, jewellery and boats), bank accounts, shares, government bonds and property annuities. Inequalities by wealth were contrasted with individual- and household measures of income and the extent to which associations were attenuated by four other measures of socioeconomic position investigated.

Relative indices of inequality (RII) were calculated for all-cause and cause-specific mortality for six different age groups (25-39, 40-54, 55-64, 65-74, 75-84, 85+ years) using Poisson regression with robust standard errors. All analyses were stratified by sex and adjusted for age (five year bands) and follow-up year.

Results Large inequalities in mortality by wealth were observed and their importance as a predictor of mortality remained more stable throughout the adult life course than income-based measures. The RII for wealth was 2.28 (95\% CI 2.17 to 2.40 ) in men aged 25-34 years compared to 5.41 (95\% CI 5.17 to $5.66)$ by individual income. Men experienced greater inequalities across all ages (e.g. the wealth-based RII was 1.82 (95\% CI 1.72 to 1.93 ) for women aged 25-34 years), except amongst the over $85 \mathrm{~s}$. Compared to individual and household income, wealth became more important over the working age period. Adjustment for education, social class, individual- and household-income led to only modest reduction in the association between wealth and mortality. Wealth was associated with nearly all causes of death and the highest relative risk increases were seen for alcohol, drugs, infections and external causes of death. Discussion Wealth is strongly predictive of mortality throughout the adult life course. Study limitations include reliance on a historical dataset and potential under-ascertainment of offshore assets (although using a rank-based measure limits its likely impact). Redistribution of income may not be sufficient to narrow health inequalities - policymakers may need to focus on the increasingly unequal distribution of wealth in high-income countries. 